\title{
Research on the Motion Control for the Under-actuated Mechanical Systems
}

\author{
Zhigang Sun \\ ${ }^{1}$ Jilin Railway Technology College, Jilin Province, China \\ a470123113@qq.com
}

Keywords: Motion Control, Under Actuated Mechanical Systems, Sliding Control

\begin{abstract}
This control of nonholonomic under actuated systems is an important field in nonlinear control. This thesis uses the example of an acrobat to illustrate the intelligent control of such systems. The acrobat is a two-link manipulator operating in a vertical plane. It consists of one joint each at the shoulder and elbow with a single actuator at the elbow. The control objective is to swing it up from a stable downward equilibrium position to the unstable straight-up equilibrium position and balance it there. To solve problems with controlling the Ac-robot, this thesis proposes intelligent control methods based on fuzzy control, variable structure control and LQR control.
\end{abstract}

\section{Introduction}

The superiority of under-actuated mechanical systems make it widely valued and objective existence, it has great advantages on saving energy enhancing the system flexibility v reducing energy consumption and the system weight than completely driven mechanical system in such aspects. From the aspects of reducing the energy consumption of systems, if the under-actuated robot can complete the full drive tasks with less drive operations, the reduction of drive will reduce the system total quality, energy consumption will also be reduced, and also it has good fault tolerance. It has better competitiveness in today's energy shortage situation, and then of course people will choose the under-actuated robot system. Under-actuated mechanical systems is a kind of nonlinear systems that generalized coordinates dimension constituting the system more than the control input dimension [1].There are different reasons for under-actuation of systems, usually are the following several kinds of situations: the system itself has some motion constraints; Robot with flexible manipulator joints and flexible operation; To increase the system's dexterity and reduce actuating devices in the design of mechanical system, etc. Generally speaking, most of the nonholonomic system belongs to the under-actuated mechanical system. Under-actuated robot of gymnastics Ac-robot is a class of second order nonholonomic constraint under-actuated mechanical systems, it is essentially a two pole robot in the vertical plane movement, has two degrees of freedom but only one drive. Ac-robot with two balance points, including vertical downward balance to stable equilibrium point, vertical balance for the unstable equilibrium point. Ac-robot control objective is to remove it from the stability of the vertical downward balance shakes a vertical unstable equilibrium point, and balance in the unstable equilibrium point [2].Because the Ac-robot dynamic constraint equation in generalized coordinates is acceleration constraints, and equation, which is different and therefore it has the second order not integrity.

An application of the under-actuated robot field is the space robot system. For robots in space or on an alien, in order to carry it to the space or an alien, we should reduce its weight as far as possible; In order for it to work for a long time, we should save energy as far as possible, a possible method is to reduce the active joints, in this way this system is composed of a class of under-actuated mechanical systems. Ac-robot as object, this paper studies the intelligent control of Ac-robot, the purpose is to investigate the second-order nonholonomic control method of under-actuated mechanical systems. Research Ac-robot such a class of under-actuated nonholonomic robot control strategy for under-actuated mechanical system, the second order the integrity system, the complex nonlinear system and the control of space robot system has extremely important significance. 


\section{The analysis and simulation of the model of Ac-robot system}

Ac-robot is a vertical plane motion of two simulation robots under-actuated mechanical systems, also known as gymnastics robot, is a kind of typical under-actuated system. It has a driver in the elbow, shoulders don't have drivers, it can do relatively complex tasks with less drive mechanism, which is widely used in nonlinear system research. Ac-robot as the research object, people launched a series of research work, because of the complexity of the Ac-robot, it is usually the movement of the space is divided into rolled up and balance, such as the Spong's teams abroad take feedback linearization method in nonlinear control theory as the theoretical basis, the system made a partial decoupling, roll up control algorithm are given, and the linear two make its stability regulator method in unstable equilibrium point. Brown and others [3] put forward several kinds of intelligent control method for Ac-robot. Lai in domestic [4] in this paper, a fuzzy variable structure control and the control strategy of combining fuzzy logic controller is used to implement fast smooth roll up control, from rolled up by fuzzy variable structure controller to ensure that quickly into the balance control. Li [5] and others take the partial feedback linearization method to design the controller, using intelligent control method to design the balance controller, two controllers by switching device to realize conversion. Zheng [6] analyzed the mechanism of discrete index near rate cause system chattering, for its lack of improved discrete reaching rate is given, and the discrete time variable structure controller is designed, and applied to the balance of the Ac-robot control. This paper studies the control of balance area; the Ac-robot system control strategy based on sliding mode control design by the interference has strong robustness. Control purpose is to stability in the vertical unstable equilibrium point of system.

Fig. 1 shows the model of Ac-robot gymnastics robot system, connecting rod connected to the base of the joints, the shoulder joint named active joints, the joints between two connecting rods named elbow is driven joints. There is only one active joint, two connecting rod under-actuated robot Ac-robot number represents a kind of active joints and passive joints is equal to the number of system, so it has certain representativeness.

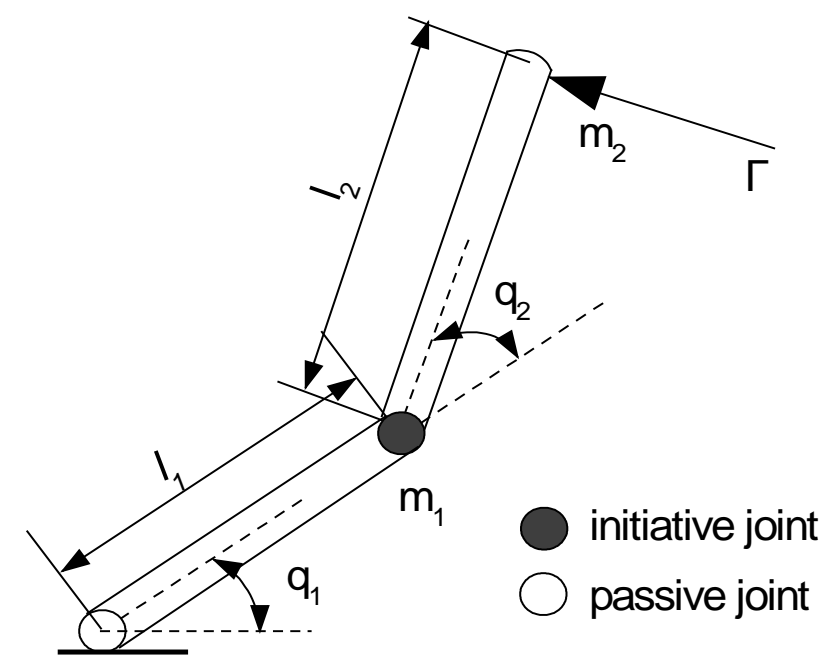

Fig.1 Acrobot system model

In the Ac-robot control, fuzzy control can ensure Ac-robot roll up quickly and then use the LQR control or fuzzy control based on TS model, can balance the Ac-robot. However, due to the Ac-robot with complex nonlinear features, and the second order non integrity, when the energy of the Ac-robot reaches the unstable equilibria of vertical potential energy, the two sticks of Ac-robot may present all sorts of attitude. When the attitude of the two stem, even if Ac-robot can reach the instability it is not balanced has potential energy, and keep the energy, also difficult to guarantee the Ac-robot by roll up area smoothly into the balance area, especially when the roll up quickly and increase the control torque, it is difficult to achieve by the roll up area to balance the rapid transition. Due to the variable 
structure controller design process itself is a process of uncoupling, using the solution convergence property of variable structure control, when fuzzy control roll up Ac-robot, can make t meet the unstable equilibria of vertical potential energy, using the fuzzy variable structure controller, the tomb of $t$ can maintain the constant at the same time, the control of the Ac-robot second pole toward relative to the first pole Angle and velocity is zero in the direction of movement, make Ac-robot two presents a natural straight, to the balance of Ac-robot easy to enter the area, from roll up area quickly transition to balance area.

This paper studies the control of balance area; the Ac-robot system control strategy based on sliding mode control design by the interference has strong robustness. Control purpose is to stability in the vertical unstable equilibrium point of system.

\section{The motion planning of the under-actuated Ac-robot motion system}

Intelligent integrated control based on fuzzy control and LQR control Ac-robot control structure is shown in Figure 2.The fuzzy controller is a fuzzy controller based on can't, including can input fuzzy, fuzzy rules and fuzzy reasoning and fuzzy solution, used to shake up the Ac-robot; When meet balance control conditions, the controller switching allows Ac-robot instruction by tomb shook up the control switch to the vagueness of the energy balance of LQR control: balance of LQR controller design according to performance index optimum target, and finally achieve the control of the Ac-robot. Fuzzy controller stable equilibrium point down from vertical roll up Ac-robot, when Ac-robot could achieve vertical unstable equilibrium point of potential energy, and after entering the balance area, the linear quadratic regulator is used to balance the Ac-robot, which is based on fuzzy control and LQR control intelligent integrated control strategy. At the back of the simulation results will prove that this control strategy control method has better performance than ever before.

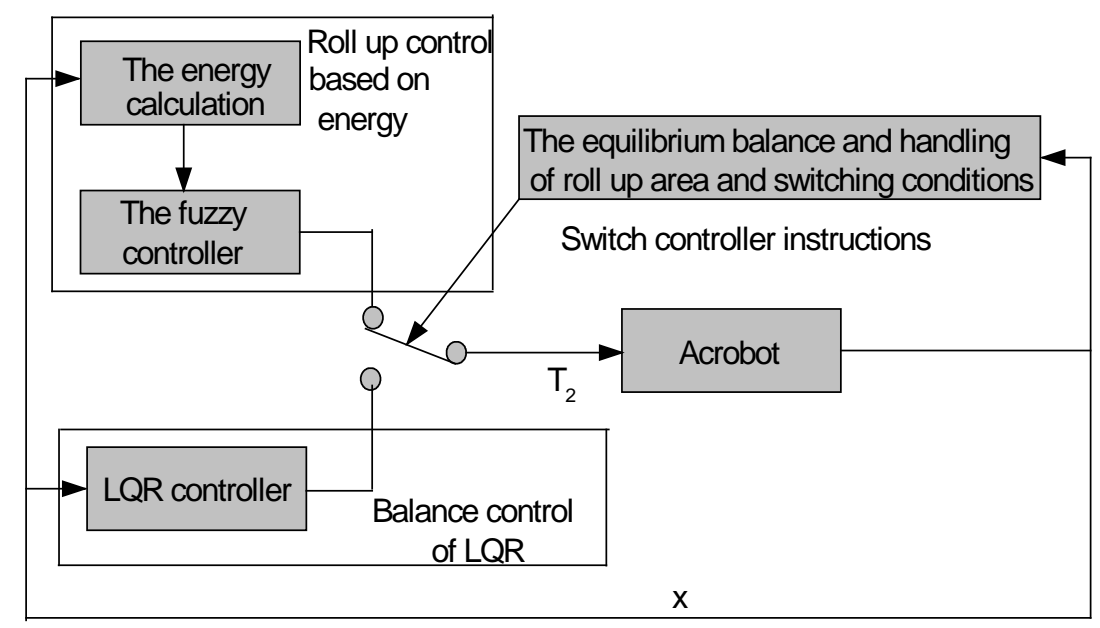

Fig.2 The control of structure Ac-robot based on fuzzy control and LQR control

For a mechanical system, its motion control problem consists of two aspects of content, one for the stability control problem, namely the designed control law, the system stability from the initial point to the target. Second for the tracking control problem, the control law is designed to make the system run along the desired trajectory, to achieve control target of the system. In general, the tracking control problem is more difficult to solve than stability control problem. In this paper, the typical two Ac-robot under-actuated mechanical systems, for example, to discuss its motion planning problem, and the problem of stability control system into the tracking control problem. First of all, the Ac-robot initial point as the vertical downward position, the position of the target as a vertical. Finally, the design makes Ac-robot along the desired trajectory tracking controller to achieve asymptotic stability. Compared with other Ac-robot stability control methods, this chapter proposed stability control method has many advantages. First, compared with IDA - PBC and other nonlinear control methods, because it will be a very complicated nonlinear system stability control problem is transformed into a 
linear time-varying error system stability control problem to solve, reduce the difficulty of controller design. Second, with the traditional partition switch than control method, it don't need to the movement of the Ac-robot space division, with only one controller can achieve the aim of stability control system, it simplifies the structure of the control system, at the same time avoid the partition control is difficult to capture Ac-robot in balance area fast and smooth. Third, because it makes the Ac-robot optimization along a path to achieve asymptotic stability, thus able to the stable motion process and the stability of the Ac-robot time to make an accurate prediction, for the selection of the drive and provides guidance in the construction of the experiment device that is unmatched by other stability control method.

\section{Conclusions}

Through the analysis of the dynamic model of Ac-robot, we reveal the three important characteristics of Ac-robot are second-order dynamic model of integrity; $t$ can be asymptotic stabilization in the time domain linear and closely near the unstable equilibrium point. On this basis, according to the characteristics of the movement of the Ac-robot proposed divided the whole motion space of the Ac-robot to shake up the area and balance area method. According to the divide of shake up area and balance area, this paper proposes an intelligent integrated control strategy based on fuzzy control and LQR control. In the roll up area, deducing based on the total energy of the Ac-robot ensure energy with each swing and increasing roll up control law, fuzzy controller is used to adjust torque control as t can increase and decrease, make the person Ac-robot by shaking up the area a smooth transition to the balance area. In the balance, the balance of the LQR controller to realize the Ac-robot control, it adopts the Ac-robot in unstable equilibrium point of linear approximation model to design. This control strategy is simple in structure; t can be solved to ensure the Ac-robot in roll up process of growing problems. Through the study of this paper has preliminarily established the under-actuated machine Ac-robot intelligent control framework, for the complete control of under-actuated mechanical systems provides a new means.

\section{Acknowledgements}

This work is supported by Independent Innovation Foundation Grant \#0118140010 to Yun Lu

\section{References}

[1] Nakamura,Y.,Integrability of dyamic constraints of underactuated mechanisms. In:The 6th International Symposium of Robotics Research, Hidden Valley, PA, October, 1993, 30-39

[2] Spong,M.W.,The swing up control problem for the acrobot.IEEE Control Systems Magazine, 1995,15(1):49-55.

[3] Brown S.C.,Passino K M. Intelligent control for an acrobot . Journal Intelligent and Robotic

Systems, 1997,18 (3):209-248.

[4] X.Z. Lai, Z.X. Cai, M. Wu. A class of under-actuated mechanical systems fuzzy and variable structure control. Journal of automation,2001,27(6):850-854.

[5] H. Li, Y.Y. Zhang. Under-actuated manipulator based on genetic algorithm optimization control research. Computer engineering and application,2006,(11):205-209.

[6] Y. Zheng, Y.W. Jing. Variable structure control of Ac-robot system based on discrete time sliding mode. Journal of northeastern university (natural science edition),2006,27(6):591-594. 